\title{
MDP on Improving Refractory Ankylosing Spondylitis Symptoms of Erythrocyte Sedimentation Rate and CRP
}

\author{
Guo Dan , Shuai Ping \\ Gannan Medical University, GanZhou 341000 China \\ 346591653@163.com
}

Keywords: MDP; ankylosing spondylitis; erythrocyte sedimentation rate; CRP; clinical efficacy

\begin{abstract}
To explore the role of MDP to improve analysis of refractory ankylosing spondylitis symptoms of erythrocyte sedimentation rate and CRP. Methods: randomly selected in December 2013 December 2014 in our hospital 60 cases of patients with ankylosing spondylitis,and divided into control and experimental groups, each group 30 cases in which the control group with oral immunosuppressants way treatment, the experimental group increased MDP treatment based on immunosuppressants, following a month of treatment,observe the patient's ESR,CRP, total efficiency improvement and therapeutic effect. The experimental group after treatment,erythrocyte sedimentation rate, and other indicators to improve the value of CRP were significantly better than the control group, the total effective rate of $96.7 \%$ in the experimental group, the total effective rate was $76.7 \%$ in the control group, a statistically difference between two groups significance $(\mathrm{P}<0.05)$. MDP for refractory ankylosing inflammation treatment has a good clinical efficacy and fewer adverse reactions, significant efficacy, worthy of clinical application.
\end{abstract}

\section{Introduction}

Ankylosing spondylitis is a clinical diagnosis and treatment of common chronic autoimmune disease,mainly in men, the spine and sacroiliac joint involvement, enthesitis is one of the main symptoms of ankylosing spondylitis,clinical symptoms of inflammatory back back pain,limited mobility, will eventually lead to patients with ankylosing bone deformities. Therefore,early treatment measures to improve the quality of life of patients is important.Currently, the clinical treatment of ankylosing spondylitis drugs including non-steroidal anti-inflammatory drugs,biologics, and drugs to improve the condition and the like,but the treatment for refractory ankylosing spondylitis are not ideal. MDP injection is a novel anti-rheumatic agents, and the trace element technetium MDP based on a combination of the drugs through the use of technetium valence electrons change gains and losses, the patient free radicals play a clear role,thereby inhibiting inflammatory mediators and the production of prostaglandins, which play an anti-inflammatory analgesic effect.Our hospital in December 2013 December 2014 were treated 60 cases of patients with ankylosing spondylitis were MDP injection treatment,and achieved satisfactory therapeutic effect, the study will now be summarized as follows:

\section{Materials and Methods}

\subsection{General Information}

Randomly selected in December 2013 December 2014 in our hospital 60 cases of ankylosing spondylitis patients for the study,and 30 patients were divided into control and experimental group of 30 patients in the control group, 22 cases of male patients, female 8 patients, aged 22 to 49 years, mean age $(30.7 \pm 6.5)$ years,duration of 1 to 27 years,mean disease duration $(10.26 \pm 8.3)$ 
years; the experimental group, 17 male patients,female 13 patients,aged 21 to 54 years old,mean age (35.6 \pm 7.9$)$ years, duration of 1 to 30 years,mean disease duration (11.3 \pm 6.6$)$ years,two groups of patients had low back pain,hip stiffness and other symptoms,and X-ray films of all patients were display varying degrees of spinal deformity.Ankylosing spondylitis is refractory means a formal non-steroidal anti-inflammatory drugs in combination at least one of methotrexate, thalidomide and other drugs more than six months of continuous treatment, the disease is still no improvement, and morning stiffness,

arthritis or disease CRP increased by $20 \%$ of cases.Basic data comparison between the two groups of patients was not statistically significant $(\mathrm{P}>0.05)$, comparable.

\subsection{Methods}

Control group:non-steroidal anti-inflammatory drugs given in combination with methotrexate or sulfasalazine tablets and other immunosuppressive therapy, usage: sulfasalazine tablets 10mg,3 times a day, continuous medication for 15 days.

Experimental group: On the basis of immunosuppressive therapy on patients administered MDP (MDP Chengdu Pharmaceutical limited liability company in which the A agent: containing technetium $0.05 \mathrm{ug}$, bottle $5 \mathrm{~mL}$; B agent: containing methylene bisacid $5 \mathrm{mg}$, stannous oxide $0.5 \mathrm{mg}$.) treatment. Usage: MDP MDP A agent B agent plus fully shake, standing 5min, 200mL of saline into the implementation of intravenous infusion, day 1, continuous infusion for 15 days.

\subsection{The clinical criteria}

According to patient outcomes to be markedly effective and ineffective three levels, which means that markedly morning stiffness symptoms disappear, lumbar activity limitation disappears, measurable indicators to improve the efficacy of ESR, CRP, and refers to the outer periphery from the number of swollen joints, etc. $\geq 70 \%$; effective means to alleviate the symptoms of morning stiffness, lumbar activity limitation eased, ESR, CRP, and refers to the measurement from the outer periphery of the efficacy index number of swollen joints and other improvement $\geq 50 \% \sim 70 \%$; invalid refers to the patient's morningstiff lumbar activity limitation and other symptoms and no improvement, ESR, CRP, refers to the distance and the number of peripheral joint swelling and other measurable indicators to improve the efficacy of $\leq 50 \%$.

\section{Statistical analysis}

Statistical analysis was performed using SPSS19.0 statistical software for data, ESR, CRP, refers to the distance and the number of peripheral joint swelling and other data indicators measured results using the average standard deviation ( $\quad$ ) to represent the mean comparison using $\mathrm{t}$ test, $\mathrm{P}$ $<0.05$ representstwo sets of data with statistical significance. 


\section{Results}

4.1Two groups of patients before and after ESR, CRP, refers to the distance and the number of peripheral joint swelling and other indicators of change.

Clinical comparative groups of patients after treatment $(\bar{X} \pm S)$

\begin{tabular}{cccccc}
\hline Group & time & ESR $(\mathrm{mm} / \mathrm{H})$ & $\mathrm{CRP}(\mathrm{mg} / \mathrm{L})$ & $\begin{array}{c}\text { It refers to the } \\
\text { distance }(\mathrm{cm})\end{array}$ & $\begin{array}{c}\text { Peripheral } \\
\text { joint swelling } \\
\text { number(cm) }\end{array}$ \\
\hline \multirow{2}{*}{ Group(30) } & Before treatment & $50.39 \pm 20.34$ & $45.62 \pm 20.04$ & $34.42 \pm 18.83$ & $7.27 \pm 5.83$ \\
& After treatment & $37.4 \pm 22.1$ & $30.95 \pm 22.64$ & $21.27 \pm 16.37$ & $3.38 \pm 1.74$ \\
test group(30) & Before treatment & $51.22 \pm 20.17$ & $44.39 \pm 21.16$ & $33.17 \pm 16.65$ & $7.05 \pm 4.72$ \\
& After treatment & $27.9 \pm 19.4$ & $18.82 \pm 17.33$ & $10.36 \pm 8.65$ & $1.26 \pm 1.02$ \\
$\mathrm{t}$ & - & 12.43 & 11.66 & 26.92 & 33.71 \\
$\mathrm{P}$ & - & 0.033 & 0.041 & 0.027 & 0.017 \\
\hline
\end{tabular}

Two groups of patients after treatment, erythrocyte sedimentation rate, CRP, refers to the distance and the number of peripheral joint swelling and other measurable indicators than before treatment improved, and the experimental group patients receiving treatment MDP, the indicators are much better than the control group, indicating MDP treatment experimental group has a better therapeutic effect, treatment effect comparison of the two groups was statistically significant difference $(\mathrm{P}<0.05)$.

\section{2 treatment groups were}

The clinical effects of two groups of patients after contrast [n (\%)]

\begin{tabular}{cccccc}
\hline Group & Number of cases & Significant effect & effective & No effect & The total efficiency \\
\hline Group & 30 & 6 & 17 & 7 & $76.7 \%$ \\
test group & 30 & 10 & 19 & 1 & $96.7 \%$ \\
$\mathrm{X}^{2}$ & - & 11.33 & 11.74 & 10.56 & 10.65 \\
$\mathrm{P}$ & - & 0.014 & 0.013 & 0.007 & 0.009 \\
\hline
\end{tabular}

In the control group six cases markedly effective in 17 cases, total effective rate was $76.7 \%$, while the experimental group, 10 cases markedly effective in 19 cases, total effective rate was $96.7 \%$, the total effective rate was significantly higher than the experimental group, two group of patients total effective contrast difference was statistically significant $(\mathrm{P}>0.05)$.

\section{Discussion}

Ankylosing spondylitis is an involvement of the spine and sacroiliac joints rheumatic diseases, early symptoms of disease usually occur sacroiliitis, lumbar disc herniation and low back myofascial inflammation go far, with arthritis the progression of the disease, the patient's spinal fusion eventually lead to normal activities gradually disappeared function, limited mobility and spinal inflammation will cause a certain impact on daily activities and quality of life of patients. Because ankylosing spondylitis cause is not yet clear, the treatment process is a long-term chronic process, and currently there is no specific drug treatment clinical emergence, the treatment of ankylosing spondylitis still need to continue to explore, if tonic spondylitis patients to take treatment early in the disease development will lay a good foundation for disease control and improved prognosis. For example, clinical diagnosis and treatment of the common diagnosis of 
ankylosing spondylitis cases, as shown below.

The patient supine hip tenderness left more obvious. CT scan showed: bilateral sacroiliac joints in patients with a small amount of damage, especially damage to the left side part of the more obvious clinical diagnosis of ankylosing spondylitis, can be combined with immunosuppressive therapy MDP, MDP morning the patient stiff, joint pain, rest pain, etc. have a role in mitigation.

Currently, clinical diagnosis and treatment drugs for the treatment of ankylosing spondylitis mainly biological agents, corticosteroids, immunosuppressive agents, and non-steroidal drugs, including MDP as a novel anti-rheumatic drugs more and more cliniciansattention by the trace element technetium MDP and MDP configuration, wherein technetium easily gains and losses in the +4 valence electron valence while maintaining the dynamic balance of nature, the removal of the patient's body through a radical change in valence, thus helping protect the superoxide dismutase activity of, and thus inhibit the production of immune complexes to exert anti-inflammatory and analgesic efficacy. The trace element technetium MDP belongs to a non-radioactive elements, methylene diphosphate salt compound belongs to a family and the ability to chelate metal ions can be formed between the two, in order to inhibit the activity of metalloproteinases effect, thereby inhibiting the destruction of bone and cartilage, and new bone formation. According to the relevant studies have shown that both cloud g anti-inflammatory and immunosuppressive effects and also inhibit bone resorption, bone repair erosion of common clinical and osteoporosis have a better therapeutic advantage.The results of this study also demonstrated MDP for the treatment of ankylosing spondylitis, two groups of patients in drug treatment administered to the patient after the acute inflammatory markers ESR, CRP etc. improved significantly, and the experimental group of patients with acute inflammatory markers erythrocyte sedimentation rate changes in CRP was significantly better than the control group, indicating MDP has a strong anti-inflammatory and analgesic effects. in addition, the overall efficiency of the experimental treatment group reached $96.7 \%$, far higher than the total efficiency, indicating yunque for the treatment of patients with ankylosing spondylitis have a more favorable clinical outcome. In summary, the MDP refractory ankylosing spondylitis have a better effect, not only can improve the quality of life of patients and reduce the psychological pressure and economic burden of patients, but also has high drug safety, MDP as the treatment of ankylosing spondylitis new drugs, significant efficacy, worthy of clinical application.

\section{References}

[1]Yong Peng, Yong Chen, Wu Di show, etc. Technetium [(99) Tc] methylene bisphosphonate treatment of refractory ankylosing spondylitis Efficacy. [J]. Chinese Health Industry, 2013, (2 ).

[2] Mei Weirong ,Liping Dong ,Honghui Wu, etc. ESR, HLA-B27 gene, C-reactive protein diagnostic value of determination of ankylosing spondylitis [J] Chinese Medicine, 2013, 10 (14): 98-100.

[3]Yongmei Ren ,Xiaoqing Zhang, Yue Wang. MDP treatment of ankylosing spondylitis Clinical Nursing of 11 cases [J] Chinese Journal of Convalescent Medicine, 2014, (2): 188-189.

[4]Nellie Hou, Yongquan Zhang, liPeng Liu, and other.MDP(99Tc- MDP) treatment of ankylosing spondylitis observation of 42 cases [J] Chinese Journal of Practical Medicine, 2013, (36): 189-190.

[5]Hongmei Zou ,Hengdong Ji,Feng Lu, etc. Evaluation. MDP Combined immunosuppressive treatment of ankylosing spondylitis [J]. Anhui Medical, 2014, (10).

[6]Jian Nie.CT and X-ray, MRI comparative study of early diagnosis of ankylosing spondylitis role of [J] Modern medicine, 2014, (3): 53-54.

[7]Suguo Chang ,Haixia Lv ,Xianbin Gao , etc. Chinese and Western medicine treatment of 
ankylosing spondylitis 19 cases [J] Chinese medicine, 2013: 324-324. 